Journal of Universal Mathematics

Vol.4 No.2 PP.188-200 (2021)

ISSN-2618-5660

DOI: $10.33773 /$ jum. 958029

\title{
ON FUNCTION SPACES CHARACTERIZED BY THE WIGNER TRANSFORM
}

\author{
ÖZNUR KULAK AND ARZU ÖMERBEYOĞLU
}

0000-0003-1433-3159 and 0000-0001-5607-2570

\begin{abstract}
Let $\omega_{i}$ be weight functions on $\mathbb{R}$, $(\mathrm{i}=1,2,3,4)$. In this work, we define $C W_{\omega_{1}, \omega_{2}, \omega_{3}, \omega_{4}}^{p, q, r, \tau}(\mathbb{R})$ to be vector space of $(f, g) \in\left(L_{\omega_{1}}^{p} \times L_{\omega_{2}}^{q}\right)(\mathbb{R})$ such that the $\tau$-Wigner transforms $W_{\tau}(f,$.$) and W_{\tau}(., g)$ belong to $L_{\omega_{3}}^{r}\left(\mathbb{R}^{2}\right)$ and $L_{\omega_{4}}^{s}\left(\mathbb{R}^{2}\right)$ respectively for $1 \leq p, q, r, s<\infty, \tau \in(0,1)$. We endow this space with a sum norm and prove that $C W_{\omega_{1}, \omega_{2}, \omega_{3}, \omega_{4}}^{p, q, r, \tau}(\mathbb{R})$ is a Banach space. We also show that $C W_{\omega_{1}, \omega_{2}, \omega_{3}, \omega_{4}}^{p, q, r, \tau}(\mathbb{R})$ becomes an essential Banach module over $\left(L_{\omega_{1}}^{1} \times L_{\omega_{2}}^{1}\right)(\mathbb{R})$. We then consider approximate identities.
\end{abstract}

\section{INTRODUCTION}

In this paper $S(\mathbb{R})$ denotes the space of complex-valued continuous functions on $\mathbb{R}$ rapidly decreasing at infinity, respectively. The space $L^{p}(\mathbb{R}),(1 \leq p<\infty)$ denotes the usual Lebesgue space. Let $\omega$ be weight function on $\mathbb{R}$, i.e., positive real valued, measurable and locally bounded function which satisfy $\omega(x) \geq 1$, $\omega(x+y) \leq \omega(x) \omega(y)$ for all $x, y \in \mathbb{R}$. For $a \geq 0$, a weight $\omega(x, t)=(1+|x|+|t|)^{a}$ which is defined on $\mathbb{R}^{2}$ is called weight of polynomial type. The weighted Lebesgue space is defined by $L_{\omega}^{p}(\mathbb{R})=\left\{f: f \omega \in L^{p}(\mathbb{R})\right\}$ for $1 \leq p<\infty$. It is known that $L_{\omega}^{p}(\mathbb{R})$ is a Banach space under the norm $\|f\|_{p, \omega}=\|f \omega\|_{p},[9]$. For any function $f$ : $\mathbb{R} \longrightarrow \mathbb{C}$, the translation, modulation and dilation operators $T_{x}, M_{\omega}$ and $D_{s}$ are given by $T_{x} f(t)=f(t-x), M_{\omega} f(t)=e^{2 \pi i \omega t} f(t)$ and $D_{s} f(t)=|s|^{-\frac{1}{2}} f\left(\frac{t}{s}\right)$ for all $x, \omega \in \mathbb{R}, 0 \neq s \in \mathbb{R}$, respectively. The parameters in wavelet theory are "time" $x$ and "scale" $s$. Dilation operator $D_{s}$ preserves the shape of $f$, but it changes the scale, [7].

Given any fixed $0 \neq g \in L^{2}(\mathbb{R})$ (called the window function), the short-time Fourier transform (STFT) of a function $f \in L^{2}(\mathbb{R})$ with respect to $g$ is defined by

$$
V_{g} f(x, \omega)=\int_{\mathbb{R}} f(t) \overline{g(t-x)} e^{-2 \pi i t \omega} d t,
$$

Date: Received: 2021-06-26; Accepted: 2021-07-28.

2000 Mathematics Subject Classification. 43A15, 43A32; 42B10.

Key words and phrases. $\tau$-Wigner transform, Essential Banach module, Approximate identity. 
for $x, \omega \in \mathbb{R}$. The short-time Fourier transform is written as convolution $V_{g} f(x, \omega)=$ $e^{-2 \pi i x \omega}\left(f * M_{\omega} g^{*}\right)(x)$, where $g^{*}(t)=\overline{g(-t)}$. It is easy to see that $V_{g} f(x, \omega)=$ $e^{-2 \pi i x \omega} V_{f} g(-x,-\omega)$. If $g$ is a compact supported function with its support centered at the origin, then the short-time Fourier transform $V_{g} f(x,$.$) is the Fourier Trans-$ form of a segment of $f$ centered in a neighborhood of $x,[7]$. Let $\tau \in(0,1)$ and let $0 \neq g \in L^{2}(\mathbb{R})$ be any fixed window function. The $\tau$-short-time Fourier transform of a function $f \in L^{2}(\mathbb{R})$ with respect to $g$ is given by $V_{g}^{\tau} f(x, \omega)=V_{g} f\left(\frac{x}{1-\tau}, \frac{\omega}{\tau}\right)$ for $x, \omega \in \mathbb{R},[1,2,10]$

The cross-Wigner distribution of $f, g \in L^{2}(\mathbb{R})$ is defined to be

$$
W(f, g)(x, \omega)=\int_{\mathbb{R}} f\left(x+\frac{t}{2}\right) \overline{g\left(x-\frac{t}{2}\right)} e^{-2 \pi i t \omega} d t
$$

for $x, \omega \in \mathbb{R}$. If $f=g$, then $W(f, f)=W f$ is said the Wigner distribution of $f \in L^{2}(\mathbb{R})$. The Wigner distribution is a quadratic time-frequency representation and it measures how much of the signal energy during the any time period which is concentrated in a frequency band. In this way, information about the energy density in the time-frequency plane is taken. It also gives the joint probability density function of the position and momentum variables, [7]. Let $\tau \in[0,1]$ and let $f, g$ be in $L^{2}(\mathbb{R})$, the $\tau$-Wigner transform is given by

$$
W_{\tau}(f, g)(x, \omega)=\int_{\mathbb{R}} f(x+\tau t) \overline{g(x-(1-\tau) t)} e^{-2 \pi i t \omega} d t, x, \omega \in \mathbb{R}
$$

$[1,2,10]$. Let $\left(X,\|\cdot\|_{X}\right)$ be a Banach space and let $\left(Y,\|\cdot\|_{Y}\right)$ be a Banach algebra. If $X$ is an algebric $Y$-module, and $\|y x\|_{X} \leq\|y\|_{Y}\|x\|_{X}$ for all $y \in Y, x \in X$, then $X$ is called a Banach $Y$-module, [12]. If a net $\left(e_{\alpha}\right)_{\alpha \in I}$ in a Banach algebra $\left(E,\|\cdot\|_{E}\right)$ satisfies $\lim _{\alpha \in I} e_{\alpha} x=x$ for all $x \in E$, then $\left(e_{\alpha}\right)_{\alpha \in I}$ is called a left approximate identity. Also if a net $\left(e_{\alpha}\right)_{\alpha \in I}$ in a Banach algebra $\left(E,\|\cdot\|_{E}\right)$ satisfies $\lim _{\alpha \in I} x e_{\alpha}=x$ for all $x \in E$, then $\left(e_{\alpha}\right)_{\alpha \in I}$ is called a right approximate identity. If a net $\left(e_{\alpha}\right)_{\alpha \in I}$ is a left approximate identity and right approximate identity, then $\left(e_{\alpha}\right)_{\alpha \in I}$ is called an approximate identity. Moreover if there exists $C>0$ such that $\left\|e_{\alpha}\right\|_{E} \leq C$ for all $\alpha \in I$, then $\left(e_{\alpha}\right)_{\alpha \in I}$ is said a bounded approximate identity, [3].

\section{Main Results}

Definition 2.1. Let $\omega_{i}(i=1,2,3,4)$ be weight functions on $\mathbb{R}$ and let $1 \leq p, q, r, s<$ $\infty, \tau \in(0,1)$. The space $C W_{\omega_{1}, \omega_{2}, \omega_{3}, \omega_{4}}^{p, q, r, s}(\mathbb{R})$ consists of all $(f, g) \in\left(L_{\omega_{1}}^{p} \times L_{\omega_{2}}^{q}\right)(\mathbb{R})$ such that their binary $\tau-$ Wigner transforms $\left(W_{\tau}(f,),. W_{\tau}(., g)\right)$ are in $\left(L_{\omega_{3}}^{r} \times L_{\omega_{4}}^{s}\right)$ $\left(\mathbb{R}^{2}\right)$. It is easy to see that

$$
\|(f, g)\|_{C W_{\omega_{1}, \omega_{2}, \omega_{3}, \omega_{4}}^{p, r, s, \tau}}=\|(f, g)\|_{L_{\omega_{1} \times L_{\omega_{2}}^{p}}^{q}}+\left\|\left(W_{\tau}(f, .), W_{\tau}(., g)\right)\right\|_{L_{\omega_{3}}^{r} \times L_{\omega_{4}}^{s}}
$$

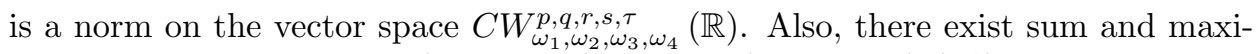
mum norms on the spaces $\left(L_{\omega_{1}}^{p} \times L_{\omega_{2}}^{q}\right)(\mathbb{R})$ and $\left(L_{\omega_{3}}^{r} \times L_{\omega_{4}}^{s}\right)\left(\mathbb{R}^{2}\right)$.

Theorem 2.2. $\left(C W_{\omega_{1}, \omega_{2}, \omega_{3}, \omega_{4}}^{p, q, r, s, \tau}(\mathbb{R}),\|\cdot\|_{C W_{\omega_{1}, \omega_{2}, \omega_{3}, \omega_{4}}^{p, q, r, s}}\right)$ is a Banach space.

Proof. Assume that $\left(\left(f_{n}, g_{n}\right)\right)_{n \in \mathbb{N}}$ is a Cauchy sequence in $C W_{\omega_{1}, \omega_{2}, \omega_{3}, \omega_{4}}^{p, q, r, \tau}(\mathbb{R})$. Clearly $\left(\left(f_{n}, g_{n}\right)\right)_{n \in \mathbb{N}}$ and $\left(\left(W_{\tau}\left(f_{n}, .\right), W_{\tau}\left(., g_{n}\right)\right)\right)_{n \in \mathbb{N}}$ are Cauchy sequences in $\left(L_{\omega_{1}}^{p} \times L_{\omega_{2}}^{q}\right)$ 
$(\mathbb{R})$ and $\left(L_{\omega_{3}}^{r} \times L_{\omega_{4}}^{s}\right)\left(\mathbb{R}^{2}\right)$, respectively. Since $\left(L_{\omega_{1}}^{p} \times L_{\omega_{2}}^{q}\right)(\mathbb{R})$ and $\left(L_{\omega_{3}}^{r} \times L_{\omega_{4}}^{s}\right)\left(\mathbb{R}^{2}\right)$ are Banach spaces, there exist $(f, g) \in\left(L_{\omega_{1}}^{p} \times L_{\omega_{2}}^{q}\right)(\mathbb{R})$ and $(h, k) \in\left(L_{\omega_{3}}^{r} \times L_{\omega_{4}}^{s}\right)\left(\mathbb{R}^{2}\right)$ such that $\left\|f_{n}-f\right\|_{p, \omega_{1}} \longrightarrow 0,\left\|g_{n}-g\right\|_{q, \omega_{2}} \longrightarrow 0,\left\|W_{\tau}\left(f_{n}, .\right)-h\right\|_{r, \omega_{3}} \longrightarrow 0$ and $\left\|W_{\tau}\left(., g_{n}\right)-k\right\|_{s, \omega_{4}} \longrightarrow 0$. This implies $\left\|W_{\tau}\left(f_{n}, .\right)-h\right\|_{r} \longrightarrow 0$ and $\left\|W_{\tau}\left(., g_{n}\right)-k\right\|_{s} \longrightarrow 0$. Then $\left(\left(W_{\tau}\left(f_{n}, .\right), W_{\tau}\left(., g_{n}\right)\right)\right)_{n \in \mathbb{N}}$ has a subsequence $\left(\left(W_{\tau}\left(f_{n_{k}}, .\right), W_{\tau}\left(., g_{n_{k}}\right)\right)\right)_{n_{k} \in \mathbb{N}}$ which converges pointwise to $(h, k)$ almost everywhere. Also it is easy to show that $\left\|f_{n_{k}}-f\right\|_{p} \longrightarrow 0$ and $\left\|g_{n_{k}}-g\right\|_{q} \longrightarrow 0$. On the other hand, if we use the Hölder inequality, then for any $u \in S(\mathbb{R})$ we find

$$
\begin{aligned}
& \left|W_{\tau}\left(f_{n_{k}}, u\right)(x, \omega)-W_{\tau}(f, u)(x, \omega)\right|= \\
& =\left|\int_{\mathbb{R}} f_{n k}(x+\tau t) \overline{u(x-(1-\tau) t)} e^{-2 \pi i \omega t} d t-\int_{\mathbb{R}} f(x+\tau t) \overline{u(x-(1-\tau) t)} e^{-2 \pi i t \omega} d t\right| \\
& \leq \int_{\mathbb{R}}\left|\left(f_{n k}-f\right)(x+\tau t) \overline{u(x-(1-\tau) t)} e^{-2 \pi i t \omega}\right| d t
\end{aligned}
$$

$$
\leq\left(\frac{1}{\tau}\right)^{\frac{1}{p}}\left(\frac{1}{1-\tau}\right)^{\frac{1}{p^{\prime}}}\left\|f_{n k}-f\right\|_{p}\|u\|_{p^{\prime}}
$$

where $\frac{1}{p}+\frac{1}{p^{\prime}}=1$. Then by $(2.1)$, we obtain

$$
\begin{aligned}
& \left|W_{\tau}(f, u)(x, \omega)-h(x, \omega)\right| \leq\left|W_{\tau}\left(f_{n_{k}}, u\right)(x, \omega)-W_{\tau}(f, u)(x, \omega)\right|+ \\
& +\left|W_{\tau}\left(f_{n_{k}}, u\right)(x, \omega)-h(x, \omega)\right| \leq \\
& \leq\left(\frac{1}{\tau}\right)^{\frac{1}{p}}\left(\frac{1}{1-\tau}\right)^{\frac{1}{p^{\prime}}}\left\|f_{n k}-f\right\|_{p}\|u\|_{p^{\prime}}+\left|W_{\tau}\left(f_{n_{k}}, .\right)(x, \omega)-h(x, \omega)\right|
\end{aligned}
$$

for any $u \in S(\mathbb{R})$. By using the inequality $(2.2)$, it is easily seen that $W_{\tau}(f,)=$. almost everywhere. So the equivalence classes of $W_{\tau}(f,$.$) and h$ are equal. Using a similar method, we find that $W_{\tau}(., g)=k$ almost everywhere. Then the equivalence classes of $W_{\tau}(., g)$ and $k$ are equal. Hence

$$
\begin{aligned}
& \left\|\left(f_{n}, g_{n}\right)-(f, g)\right\|_{C W_{\omega_{1}}^{p, q, r, s, \omega_{2}, \omega_{3}, \omega_{4}}}=\left\|\left(f_{n}-f, g_{n}-g\right)\right\|_{L_{\omega_{1}}^{p} \times L_{\omega_{2}}^{q}}+ \\
& +\left\|\left(W_{\tau}\left(f_{n}-f, .\right), W_{\tau}\left(., g_{n}-g\right)\right)\right\|_{L_{\omega_{3}}^{r} \times L_{\omega_{4}}^{s}} \longrightarrow 0
\end{aligned}
$$

and $(f, g) \in C W_{\omega_{1}, \omega_{2}, \omega_{3}, \omega_{4}}^{p, q, r, s, \tau}(\mathbb{R})$. That means $C W_{\omega_{1}, \omega_{2}, \omega_{3}, \omega_{4}}^{p, q, r, s, \tau}(\mathbb{R})$ is a Banach space.

Theorem 2.3. Let $\omega_{i}(i=1,2,3,4)$ be weight functions of polynomial type. Then $(S \times S)(\mathbb{R})$ is dense in $C W_{\omega_{1}, \omega_{2}, \omega_{3}, \omega_{4}}^{p, q, r, \tau}(\mathbb{R})$.

Proof. Take any $(f, g) \in(S \times S)(\mathbb{R})$. Then $\left(W_{\tau}(f,),. W_{\tau}(., g)\right) \in(S \times S)\left(\mathbb{R}^{2}\right)$. Since $\omega_{i}(i=1,2,3,4)$ are weight functions of polynomial type, we have $(f, g) \in$ $\left(L_{\omega_{1}}^{p} \times L_{\omega_{2}}^{q}\right)(\mathbb{R})$ and $\left(W_{\tau}(f,),. W_{\tau}(., g)\right) \in\left(L_{\omega_{3}}^{r} \times L_{\omega_{4}}^{s}\right)\left(\mathbb{R}^{2}\right)$. That means $(f, g) \in$ $C W_{\omega_{1}, \omega_{2}, \omega_{3}, \omega_{4}}^{p, q, r, s}(\mathbb{R})$. Hence we have $(S \times S)(\mathbb{R}) \subset C W_{\omega_{1}, \omega_{2}, \omega_{3}, \omega_{4}}^{p, q, r, s}(\mathbb{R})$.

Now take any $(f, g) \in C W_{\omega_{1}, \omega_{2}, \omega_{3}, \omega_{4}}^{p, q, s, \tau}(\mathbb{R})$. Then we have $(f, g) \in\left(L_{\omega_{1}}^{p} \times L_{\omega_{2}}^{q}\right)(\mathbb{R})$ and $\left(W_{\tau}(f,),. W_{\tau}(., g)\right) \in\left(L_{\omega_{3}}^{r} \times L_{\omega_{4}}^{s}\right)\left(\mathbb{R}^{2}\right)$. Since $\overline{(S \times S)(\mathbb{R})}=\left(L_{\omega_{1}}^{p} \times L_{\omega_{2}}^{q}\right)(\mathbb{R})$ and $\overline{(S \times S)\left(\mathbb{R}^{2}\right)}=\left(L_{\omega_{3}}^{r} \times L_{\omega_{4}}^{s}\right)\left(\mathbb{R}^{2}\right)$, there exist $\left(\left(h_{n}, k_{n}\right)\right)_{n \in \mathbb{N}} \subset(S \times S)(\mathbb{R})$ and 
$\left(\left(H_{n}, K_{n}\right)\right)_{n \in \mathbb{N}} \subset(S \times S)\left(\mathbb{R}^{2}\right)$ such that

$$
\left\|(f, g)-\left(h_{n}, k_{n}\right)\right\|_{L_{\omega_{1}}^{p} \times L_{\omega_{2}}^{q}} \rightarrow 0
$$

and

$$
\left\|\left(W_{\tau}(f, .), W_{\tau}(., g)\right)-\left(H_{n}, K_{n}\right)\right\|_{L_{\omega_{3}}^{r} \times L_{\omega_{4}}^{s}} \rightarrow 0 .
$$

Then by (2.4), we have $\left\|W_{\tau}(f, .)-H_{n}\right\|_{r} \rightarrow 0$ and $\left\|W_{\tau}(., g)-K_{n}\right\|_{s} \rightarrow 0$. So $\left(H_{n}\right)_{n \in \mathbb{N}}$ and $\left(K_{n}\right)_{n \in \mathbb{N}}$ have subsequences $\left(H_{n_{k}}\right)_{n_{k} \in \mathbb{N}}$ and $\left(K_{n_{k}}\right)_{n_{k} \in \mathbb{N}}$ which converge pointwise to $W_{\tau}(f,$.$) and W_{\tau}(., g)$ almost everywhere, respectively. Then, we easily show that

$$
\left\|W_{\tau}(f, .)-H_{n_{k}}\right\|_{r, \omega_{3}} \rightarrow 0,\left\|W_{\tau}(., g)-K_{n_{k}}\right\|_{s, \omega_{4}} \rightarrow 0 .
$$

Using Hölder inequality, we have for any $u \in S(\mathbb{R})$

$$
\begin{aligned}
& \left|W_{\tau}\left(h_{n}, u\right)(x, \omega)-H_{n_{k}}(x, \omega)\right| \leq\left|W_{\tau}(f, u)(x, \omega)-H_{n_{k}}(x, \omega)\right|+ \\
& +\left|W_{\tau}(f, u)(x, \omega)-W_{\tau}\left(h_{n}, u\right)(x, \omega)\right| \\
& \leq\left|W_{\tau}(f, u)(x, \omega)-H_{n_{k}}(x, \omega)\right|+\int_{\mathbb{R}}\left|\left(f-h_{n}\right)(x+\tau t)\right||u(x-(1-\tau) t)| d t
\end{aligned}
$$

$$
\leq\left|W_{\tau}(f, u)(x, \omega)-H_{n_{k}}(x, \omega)\right|+\left(\frac{1}{\tau}\right)^{\frac{1}{p}}\left(\frac{1}{1-\tau}\right)^{\frac{1}{p^{\prime}}}\left\|f-h_{n}\right\|_{p}\|u\|_{p^{\prime}},
$$

where $\frac{1}{p}+\frac{1}{p^{\prime}}=1$. By $(2.3)$ and $(2.6)$, we achieve $W_{\tau}\left(h_{n},.\right)=H_{n_{k}}$. Similarly, we can write $W_{\tau}\left(., k_{n}\right)=K_{n_{k}}$. Then by $(2.5)$, we find

$$
\left\|W_{\tau}(f, .)-W_{\tau}\left(h_{n}, .\right)\right\|_{r, \omega_{3}} \rightarrow 0,\left\|W_{\tau}(., g)-W_{\tau}\left(., k_{n}\right)\right\|_{s, \omega_{4}} \rightarrow 0 .
$$

This implies

$$
\left\|\left(W_{\tau}(f, .), W_{\tau}(., g)\right)-\left(W_{\tau}\left(h_{n}, .\right), W_{\tau}\left(., k_{n}\right)\right)\right\|_{L_{\omega_{3}}^{r} \times L_{\omega_{4}}^{s}} \rightarrow 0 .
$$

Finally combining (2.3) and (2.7), we get

$$
\begin{aligned}
& \left\|(f, g)-\left(h_{n}, k_{n}\right)\right\|_{C W_{\omega_{1}, \omega_{2}, \omega_{3}, \omega_{4}}^{p, r, s, \tau}}=\left\|(f, g)-\left(h_{n}, k_{n}\right)\right\|_{L_{\omega_{1} \times L_{\omega_{2}}^{p}}^{q}+}+ \\
+ & \left\|\left(W_{\tau}(f, .), W_{\tau}(., g)\right)-\left(W_{\tau}\left(h_{n}, .\right), W_{\tau}\left(., k_{n}\right)\right)\right\|_{L_{\omega_{3}}^{r} \times L_{\omega_{4}}^{s}} \longrightarrow 0 .
\end{aligned}
$$

Therefore the proof is completed.

Definition 2.4. Let $\omega_{1}$ and $\omega_{3}$ be weight functions on $\mathbb{R}$ and let be $1 \leq p, r<\infty$, $\tau \in(0,1)$. The space $C W_{\omega_{1}, \omega_{3}}^{p, r, \tau}(\mathbb{R})$ consists of all $f \in L_{\omega_{1}}^{p}(\mathbb{R})$ such that their $\tau$-Wigner transforms $W_{\tau}(f,$.$) are in L_{\omega_{3}}^{r}\left(\mathbb{R}^{2}\right)$. We endow this space with the sum norm

$$
\|f\|_{C W_{\omega_{1}, \omega_{3}}^{p, r, \tau}}=\|f\|_{p, \omega_{1}}+\left\|W_{\tau}(f, .)\right\|_{r, \omega_{3}} .
$$

Let $\omega_{2}$ and $\omega_{4}$ be weight functions on $\mathbb{R}$ and let be $1 \leq q, s<\infty, \tau \in(0,1)$. The space $C W_{\omega_{2}, \omega_{4}}^{q, s, \tau}(\mathbb{R})$ consists of all $g \in L_{\omega_{2}}^{q}(\mathbb{R})$ such that their $\tau$-Wigner transforms $W_{\tau}(., g)$ are in $L_{\omega_{4}}^{s}\left(\mathbb{R}^{2}\right)$. This space is equipped with the sum norm

$$
\|g\|_{C W_{\omega_{2}, \omega_{4}}^{q, s, \tau}}=\|g\|_{q, \omega_{2}}+\left\|W_{\tau}(., g)\right\|_{s, \omega_{4}} .
$$

By using the method in Theorem 1, it is easy to see that this spaces $C W_{\omega_{1}, \omega_{3}}^{p, r, \tau}(\mathbb{R})$ and $C W_{\omega_{2}, \omega_{4}}^{q, s, \tau}(\mathbb{R})$ are Banach space with these sum norm.

Lemma 2.5. The space $C W_{\omega_{1}, \omega_{2}, \omega_{3}, \omega_{4}}^{p, q, r, \tau}(\mathbb{R})$ is isomorphic to $\left(C W_{\omega_{1}, \omega_{3}}^{p, r, \tau} \times C W_{\omega_{2}, \omega_{4}}^{q, s, \tau}\right)(\mathbb{R})$. 
Proof. Take the mapping $I: C W_{\omega_{1}, \omega_{2}, \omega_{3}, \omega_{4}}^{p, q, r, s, \tau}(\mathbb{R}) \rightarrow\left(C W_{\omega_{1}, \omega_{3}}^{p, r, \tau} \times C W_{\omega_{2}, \omega_{4}}^{q, s, \tau}\right)(\mathbb{R}), I((f, g))$ $=(f, g)$. It is clear that this mapping is linear and bijective. Also, since

$$
\begin{aligned}
& \|H((f, g))\|_{C W_{\omega_{1}, \omega_{2}, \omega_{3}, \omega_{4}}^{p, q, r, \tau},}=\|(f, g)\|_{L_{\omega_{1}}^{p} \times L_{\omega_{2}}^{q}}+\left\|\left(W_{\tau}(f, .), W_{\tau}(., g)\right)\right\|_{L_{\omega_{3}}^{r} \times L_{\omega_{4}}^{s}} \\
& =\|f\|_{p, \omega_{1}}+\|g\|_{q, \omega_{2}}+\left\|W_{\tau}(f, .)\right\|_{r, \omega_{3}}+\left\|W_{\tau}(., g)\right\|_{s, \omega_{4}} \\
& =\|f\|_{p, \omega_{1}}+\left\|W_{\tau}(f, .)\right\|_{r, \omega_{3}}+\|g\|_{q, \omega_{2}}+\left\|W_{\tau}(., g)\right\|_{s, \omega_{4}} \\
& =\|f\|_{C W_{\omega_{1}, \omega_{3}}^{p, r, \tau}}+\|g\|_{C W_{\omega_{2}, \omega_{4}}^{q, s, \tau}}=\|(f, g)\|_{C W_{\omega_{1}, \omega_{3}}^{p, r, \tau} \times C W_{\omega_{2}, \omega_{4}, \tau}^{q, \tau}},
\end{aligned}
$$

The mapping $I$ is isometry of $C W_{\omega_{1}, \omega_{2}, \omega_{3}, \omega_{4}}^{p, q, r, s}(\mathbb{R})$ into $\left(C W_{\omega_{1}, \omega_{3}}^{p, r, \tau} \times C W_{\omega_{2}, \omega_{4}}^{q, s, \tau}\right)(\mathbb{R})$. Therefore, we obtain that $C W_{\omega_{1}, \omega_{2}, \omega_{3}, \omega_{4}}^{p, q, r, \tau}(\mathbb{R}) \cong\left(C W_{\omega_{1}, \omega_{3}}^{p, r, \tau} \times C W_{\omega_{2}, \omega_{4}}^{q, s, \tau}\right)(\mathbb{R})$.

Definition 2.6. Let $f$ and $g$ be any functions on $\mathbb{R}$. The binary translation mapping is defined by

$$
T_{x}(f, g)(t)=\left(T_{x} f(t), T_{x} g(t)\right)=(f(t-x), g(t-x)), x, t \in \mathbb{R} .
$$

The following lemma is written easily from Proposition4 in [11]

Lemma 2.7. For $\tau \in(0,1)$ and $z \in \mathbb{R}$, we have

$$
W_{\tau}\left(T_{z} f, h\right)(x, \omega)=e^{-2 \pi i \omega z} T_{(z(1-\tau), 0)} W_{\tau}(f, h)(x, \omega)
$$

and

$$
W_{\tau}\left(k, T_{z} g\right)(x, \omega)=e^{2 \pi i \omega z} T_{(z \tau, 0)} W_{\tau}(k, g) .
$$

Theorem 2.8. Assume that $\omega_{3}$ is symmetric weight function. The space $C W_{\omega_{1}, \omega_{2}, \omega_{3}, \omega_{4}}^{p, q, r, s, \tau}$ is invariant under binary translations. Moreover,

$$
\left\|T_{z}(f, g)\right\|_{C W_{\omega_{1}, \omega_{2}, \omega_{3}, \omega_{4}}^{p, q, r, s, \tau}} \leq(u(z)+v((z, 0)) v((z \tau, 0)))\|(f, g)\|_{C W_{\omega_{1}, \omega_{2}, \omega_{3}, \omega_{4}}^{p, q, r, \tau},},
$$

where $u=\max \left\{\omega_{1}, \omega_{2}\right\}$ and $v=\max \left\{\omega_{3}, \omega_{4}\right\}$.

Proof. Let $(f, g) \in C W_{\omega_{1}, \omega_{2}, \omega_{3}, \omega_{4}}^{p, q, r, \tau}(\mathbb{R})$. Then, we write $(f, g) \in\left(L_{\omega_{1}}^{p} \times L_{\omega_{2}}^{q}\right)(\mathbb{R})$ and $\left(W_{\tau}(f,),. W_{\tau}(., g)\right) \in\left(L_{\omega_{3}}^{r} \times L_{\omega_{4}}^{s}\right)\left(\mathbb{R}^{2}\right)$. Also, since $\left\|T_{z} f\right\|_{p, \omega_{1}} \leq \omega_{1}(z)\|f\|_{p, \omega_{1}}$ and $\left\|T_{z} g\right\|_{q, \omega_{2}} \leq \omega_{2}(z)\|f\|_{q, \omega_{2}}[5]$, we have

$$
\begin{aligned}
& \left\|T_{z}(f, g)\right\|_{L_{\omega_{1} \times L_{\omega_{2}}^{q}}^{q}}=\left\|T_{z} f\right\|_{p, \omega_{1}}+\left\|T_{z} g\right\|_{q, \omega_{2}} \\
& \leq \omega_{1}(z)\|f\|_{p, \omega_{1}}+\omega_{2}(z)\|f\|_{q, \omega_{2}} \\
& \leq u(z)\|(f, g)\|_{L_{\omega_{1}}^{p} \times L_{\omega_{2}}^{q}},
\end{aligned}
$$

where $u=\max \left\{\omega_{1}, \omega_{2}\right\}$. Then, we write $T_{z}(f, g) \in\left(L_{\omega_{1}}^{p} \times L_{\omega_{2}}^{q}\right)(\mathbb{R})$ for all $z \in \mathbb{R}$. By Lemma 2.7, we have

$$
\begin{aligned}
& \left\|\left(W_{\tau}\left(T_{z} f, .\right), W_{\tau}\left(., T_{z} g\right)\right)\right\|_{L_{\omega_{3}}^{r} \times L_{\omega_{4}}^{s}}=\left\|W_{\tau}\left(T_{z} f, .\right)\right\|_{r, \omega_{3}}+\left\|W_{\tau}\left(., T_{z} g\right)\right\|_{s, \omega_{4}} \\
& =\left\|e^{-2 \pi i \omega z} T_{(z(1-\tau), 0)} W_{\tau}(f, .)\right\|_{r, \omega_{3}}+\left\|e^{2 \pi i \omega z} T_{(z \tau, 0)} W_{\tau}(., g)\right\|_{s, \omega_{4}} \\
& \leq \omega_{3}((z(1-\tau), 0))\left\|W_{\tau}(f, .)\right\|_{r, \omega_{3}}+\omega_{4}((z \tau, 0))\left\|W_{\tau}(., g)\right\|_{s, \omega_{4}} \\
& \leq \omega_{3}((z, 0)) \omega_{3}((z \tau, 0))\left\|W_{\tau}(f, .)\right\|_{r, \omega_{3}}+\omega_{4}((z, 0)) \omega_{4}((z \tau, 0))\left\|W_{\tau}(., g)\right\|_{s, \omega_{4}} \\
& \leq v((z, 0)) v((z \tau, 0))\left\|\left(W_{\tau}(f, .), W_{\tau}(., g)\right)\right\|_{L_{\omega_{3}}^{r} \times L_{\omega_{4}}^{s}},
\end{aligned}
$$


where $v=\max \left\{\omega_{3}, \omega_{4}\right\}$. Combining (2.8) and (2.9),

$$
\begin{aligned}
& \left\|T_{z}(f, g)\right\|_{C W_{\omega_{1}, \omega_{2}, \omega_{3}, \omega_{4}}^{p, q, s, \tau}}=\left\|\left(T_{z} f, T_{z} g\right)\right\|_{L_{\omega_{1}}^{p} \times L_{\omega_{2}}^{q}}+\left\|\left(W_{\tau}\left(T_{z} f, .\right), W_{\tau}\left(., T_{z} g\right)\right)\right\|_{L_{\omega_{3}}^{r} \times L_{\omega_{4}}^{s}} \\
& \leq u(z)\|(f, g)\|_{L_{\omega_{1}}^{p} \times L_{\omega_{2}}^{q}}^{q}+v((z, 0)) v((z \tau, 0))\left\|\left(W_{\tau}(f, .), W_{\tau}(., g)\right)\right\|_{L_{\omega_{3}}^{r} \times L_{\omega_{4}}^{s}} \\
& \leq u(z)\|(f, g)\|_{\left.C W_{\omega_{1}, \omega_{2}, \omega_{3}, \omega_{4}}^{p, q, r, s, \tau}\right)}+v((z, 0)) v((z \tau, 0))\|(f, g)\|_{C W_{\omega_{1}, \omega_{2}, \omega_{3}, \omega_{4}}^{p, q, r, s,}} \\
& =(u(z)+v((z, 0)) v((z \tau, 0)))\|(f, g)\|_{C W_{\omega_{1}, \omega_{2}, \omega_{3}, \omega_{4}}^{p, q, r, s}} .
\end{aligned}
$$

Finally, we say $T_{z}(f, g) \in C W_{\omega_{1}, \omega_{2}, \omega_{3}, \omega_{4}}^{p, q, r, \tau}(\mathbb{R})$.

Definition 2.9. Let $f, g, h, k$ be Borel measurable functions on $\mathbb{R}$. The binary convolution is defined by $(f, g) *(h, k)=(f * h, g * k)$, where "*" denotes usual convolution. The following conditions must be required for the binary convolution to be defined;

$$
\int_{\mathbb{R}}|f(y) h(x-y)| d y<\infty
$$

and

$$
\int_{\mathbb{R} .}|g(y) k(x-y)| d y<\infty
$$

Theorem 2.10. a)Assume that $\omega_{3}$ is symmetric weight function. The binary translation mapping $(f, g) \longrightarrow T_{z}(f, g)$ is continuous from $C W_{\omega_{1}, \omega_{2}, \omega_{3}, \omega_{4}}^{p, q, r, s,}(\mathbb{R})$ into $C W_{\omega_{1}, \omega_{2}, \omega_{3}, \omega_{4}}^{p, q, s, \tau}(\mathbb{R})$ for every fixed $z \in \mathbb{R}$.

b) The binary translation mapping $z \rightarrow T_{z}(f, g)$ is continuous from $\mathbb{R}$ into $C W_{\omega_{1}, \omega_{2}, \omega_{3}, \omega_{4}}^{p, q, s, s}(\mathbb{R})$.

Proof. a) Let $(f, g) \in C W_{\omega_{1}, \omega_{2}, \omega_{3}, \omega_{4}}^{p, q, s, \tau}(\mathbb{R})$ be given. It is enough to prove the theorem for $(f, g)=(0,0)$. Let $\varepsilon>0$ be given. Choose an $\delta>0$ such that $\delta=\frac{\varepsilon}{u(z)+v((z, 0)) v((z \tau, 0))}$. Thus, if $\|(f, g)\|_{C W_{\omega_{1}, \omega_{2}, \omega_{3}, \omega_{4}}^{p, q, r, \tau}, \omega_{4}}<\delta$, then by $(2.9)$

$$
\begin{aligned}
\left\|T_{z}(f, g)\right\|_{C W_{\omega_{1}, \omega_{2}, \omega_{3}, \omega_{4}}^{p, q, r, s, \tau}} & \leq(u(z)+v((z, 0)) v((z \tau, 0)))\|(f, g)\|_{C W_{\omega_{1}, \omega_{2}, \omega_{3}, \omega_{4}}^{p, q, r, \tau}} \\
& <\delta(u(z)+v((z, 0)) v((z \tau, 0)))=\varepsilon .
\end{aligned}
$$

b) Take any $(f, g) \in C W_{\omega_{1}, \omega_{2}, \omega_{3}, \omega_{4}}^{p, q, r, s}(\mathbb{R})$. It is known that the translation mapping is continuous from $\mathbb{R}$ into $L_{\omega_{1}}^{p}(\mathbb{R})$ and $L_{\omega_{2}}^{q}(\mathbb{R})$, [5]. So for any given $\varepsilon>0$, there exists $\delta_{1}(\varepsilon)>0$ such that if $|z-u|<\delta_{1}$ for $z, u \in \mathbb{R}$, then

$$
\begin{aligned}
\left\|T_{z}(f, g)-T_{u}(f, g)\right\|_{L_{\omega_{1}}^{p} \times L_{\omega_{2}}^{q}} & =\left\|\left(T_{z} f-T_{u} f-, T_{z} g-T_{u} g\right)\right\|_{L_{\omega_{1}}^{p} \times L_{\omega_{2}}^{q}} \\
& =\max \left\{\left\|T_{z} f-T_{u} f\right\|_{p, \omega_{1}},\left\|T_{z} g-T_{u} g\right\|_{q, \omega_{2}}\right\}<\frac{\varepsilon}{2}
\end{aligned}
$$

Also since the modulation mapping is continuous from $\mathbb{R}$ into $L_{\omega_{3}}^{r}\left(\mathbb{R}^{2}\right)$ and $L_{\omega_{4}}^{s}\left(\mathbb{R}^{2}\right)$ [5], for the same $\varepsilon>0$, there exists $\delta_{2}(\varepsilon)>0$ such that if $|z-u|<\delta_{2}$ for $z, u \in \mathbb{R}$, 
then

$$
\begin{aligned}
& \left\|\left(W_{\tau}\left(T_{z} f-T_{u} f, .\right), W_{\tau}\left(., T_{z} g-T_{u} g\right)\right)\right\|_{L_{\omega_{3}}^{r} \times L_{\omega_{4}}^{s}}= \\
& =\left\|W_{\tau}\left(T_{z} f-T_{u} f, .\right)\right\|_{r, \omega_{3}}+\left\|W_{\tau}\left(., T_{z} g-T_{u} g\right)\right\|_{s, \omega_{4}}= \\
& =\max \left\{\left\|e^{-2 \pi i \omega z} T_{(z(1-\tau), 0)} W_{\tau}(f, .)-e^{-2 \pi i \omega u} T_{(u(1-\tau), 0)} W_{\tau}(f, .)\right\|_{r, \omega_{3}},\right. \\
& \left.\left\|e^{2 \pi i \omega z} T_{(z \tau, 0)} W_{\tau}(., g)-e^{2 \pi i \omega u} T_{(u \tau, 0)} W_{\tau}(., g)\right\|_{s, \omega_{4}}\right\}= \\
& =\max \left\{\left\|M_{(0,-z)} T_{(z(1-\tau), 0)} W_{\tau}(f, .)-M_{(0,-u)} T_{(u(1-\tau), 0)} W_{\tau}(f, .)\right\|_{r, \omega_{3}},\right. \\
& \left.\left\|M_{(0, z)} T_{(z \tau, 0)} W_{\tau}(., g)-M_{(0, u)} T_{(u \tau, 0)} W_{\tau}(., g)\right\|_{s, \omega_{4}}\right\}<\frac{\varepsilon}{2}
\end{aligned}
$$

Set $\delta=\min \left\{\delta_{1}, \delta_{2}\right\}$. From (2.10) and (2.11), if $|z-u|<\delta$ for $z, u \in \mathbb{R}$, then

$$
\begin{aligned}
& \left\|T_{z}(f, g)-T_{u}(f, g)\right\|_{C W_{\omega_{1}, \omega_{2}, \omega_{3}, \omega_{4}}^{p, q, r, \tau}}=\left\|\left(T_{z} f-T_{u} f-, T_{z} g-T_{u} g\right)\right\|_{C W_{\omega_{1}, \omega_{2}, \omega_{3}, \omega_{4}}^{p, q, r, s}, \omega_{4}} \\
& =\left\|\left(T_{z} f-T_{u} f-, T_{z} g-T_{u} g\right)\right\|_{L_{\omega_{1}}^{p} \times L_{\omega_{2}}^{q}}^{q}+ \\
& +\left\|\left(W_{\tau}\left(T_{z} f-T_{u} f, .\right), W_{\tau}\left(., T_{z} g-T_{u} g\right)\right)\right\|_{L_{\omega_{3}}^{r} \times L_{\omega_{4}}^{s}}<\frac{\varepsilon}{2}+\frac{\varepsilon}{2}=\varepsilon .
\end{aligned}
$$

Corollary 1. a) The binary translation mapping $z \rightarrow T_{z}(f, g)$ is continuous from $\mathbb{R}$ into $C W_{\omega_{1}, \omega_{3}}^{p, r, \tau}(\mathbb{R})$.

b) The binary translation mapping $z \rightarrow T_{z}(f, g)$ is continuous from $\mathbb{R}$ into $C W_{\omega_{2}, \omega_{4}}^{q, s, \tau}(\mathbb{R})$.

Lemma 2.11. Let $f, g \in S(\mathbb{R})$. If $\tau \in(0,1)$, then

$$
W_{\tau}(f, g)(x, \omega)=e^{\frac{2 \pi i x \omega}{\tau}} \frac{1}{\sqrt{\tau(1-\tau)}} V_{D_{\bar{\tau}-1}^{\tau}} g f(x, \omega)
$$

holds for all $x, \omega \in \mathbb{R}$.

Proof. Assume that $f, g \in S(\mathbb{R})$. If we make the substitution $u=x+\tau t$, then we have

$$
\begin{aligned}
& W_{\tau}(f, g)(x, \omega)=\int_{\mathbb{R}} f(x+\tau t) \overline{g(x-(1-\tau) t)} e^{-2 \pi i \omega t} d t \\
& =\int_{\mathbb{R}} f(u) \overline{g\left(u-\frac{u-x}{\tau}\right)} e^{-2 \pi i \omega\left(\frac{u-x}{\tau}\right)} \frac{d u}{\tau} \\
& =\int_{\mathbb{R}} f(u) \overline{g\left(u\left(\frac{\tau-1}{\tau}\right)+\frac{x}{\tau}\right)} e^{-2 \pi i \omega\left(\frac{u-x}{\tau}\right)} \frac{d u}{\tau} \\
& =\frac{1}{\tau} e^{2 \pi i \frac{x \omega}{\tau}} \int_{\mathbb{R}} f(u) \overline{g\left(\left(\frac{\tau-1}{\tau}\right)\left(u-\frac{x}{1-\tau}\right)\right)} e^{-2 \pi i \frac{\omega u}{\tau}} d u \\
& =\frac{1}{\tau} e^{2 \pi i \frac{x \omega}{\tau}}\left|\frac{\tau}{\tau-1}\right|^{\frac{1}{2}} \int_{\mathbb{R}} f(u) \overline{D_{\frac{\tau}{\tau-1}} g\left(u-\frac{x}{1-\tau}\right)} e^{-2 \pi i \frac{\omega u}{\tau}} d u \\
& =e^{\frac{2 \pi i x \omega}{\tau}} \frac{1}{\sqrt{\tau(1-\tau)}} V_{D_{\frac{\tau}{\tau-1}}^{\tau} g} f(x, \omega)
\end{aligned}
$$

for all $x, \omega \in \mathbb{R}$. 
Theorem 2.12. Let $f, h, g, k, f_{1}, f_{2} \in S(\mathbb{R})$. If $\tau \in(0,1)$, then

$$
W_{\tau}\left(f * h, f_{1}\right)(x, \omega)=\frac{1}{\sqrt{\tau(1-\tau)}} e^{\frac{-2 \pi i x \omega}{1-\tau}}\left(h *\left(f * M_{\frac{\omega}{\tau}}\left(D_{\frac{\tau}{\tau-1}} f_{1}\right)^{*}\right)\right)\left(\frac{x}{1-\tau}\right)
$$

and

$$
W_{\tau}\left(f_{2}, g * k\right)(x, \omega)=\frac{1}{\tau} e^{\frac{2 \pi i x \omega(1+\tau)}{\tau(1-\tau)}} \overline{\left(D_{\frac{\tau}{\tau-1}} k *\left(D_{\bar{\tau}-1} g * M_{\frac{-\omega}{\tau}} f_{2}^{*}\right)\right)\left(\frac{-x}{1-\tau}\right)}
$$

holds for all $x, \omega \in \mathbb{R}$.

Proof. Take any $f, h, f_{1} \in S(\mathbb{R})$. Then by Lemma 2.11, we have

$$
\begin{aligned}
& W_{\tau}\left(f * h, f_{1}\right)(x, \omega)=e^{\frac{2 \pi i x \omega}{\tau}} \frac{1}{\sqrt{\tau(1-\tau)}} V_{D_{\frac{\tau}{\tau-1}}^{\tau} f_{1}}(f * h)(x, \omega) \\
& =e^{\frac{2 \pi i x \omega}{\tau}} \frac{1}{\sqrt{\tau(1-\tau)}} V_{D_{\frac{\tau}{\tau-1}}} f_{1}(f * h)\left(\frac{x}{1-\tau}, \frac{\omega}{\tau}\right) \\
& =e^{\frac{2 \pi i x \omega}{\tau}} \frac{1}{\sqrt{\tau(1-\tau)}} e^{\frac{-2 \pi i x \omega}{\tau(1-\tau)}}\left((f * h) * M_{\frac{\omega}{\tau}}\left(D_{\frac{\tau}{\tau-1}} f_{1}\right)^{*}\right)\left(\frac{x}{1-\tau}\right) \\
& =\frac{1}{\sqrt{\tau(1-\tau)}} e^{\frac{-2 \pi i x \omega}{1-\tau}}\left(h *\left(f * M_{\frac{\omega}{\tau}}\left(D_{\frac{\tau}{\tau-1}} f_{1}\right)^{*}\right)\right)\left(\frac{x}{1-\tau}\right) .
\end{aligned}
$$

Now take any $g, k, f_{2} \in S(\mathbb{R})$. Again by Lemma 2.11, we get

$$
\begin{aligned}
& W_{\tau}\left(f_{2}, g * k\right)(x, \omega)=e^{\frac{2 \pi i x \omega}{\tau}} \frac{1}{\sqrt{\tau(1-\tau)}} V_{D_{\frac{\tau}{\tau-1}}^{\tau}}(g * k) f_{2}(x, \omega) \\
& =e^{\frac{2 \pi i x \omega}{\tau}} \frac{1}{\sqrt{\tau(1-\tau)}} V_{D_{\frac{\tau}{\tau-1}}(g * k)} f_{2}\left(\frac{x}{1-\tau}, \frac{\omega}{\tau}\right) \\
& =e^{\frac{2 \pi i x \omega}{\tau}} \frac{1}{\sqrt{\tau(1-\tau)}} e^{\frac{-2 \pi i x \omega}{\tau(1-\tau)}} V_{f_{2}} D_{\frac{\tau}{\tau-1}}(g * k)\left(\frac{-x}{1-\tau}, \frac{-\omega}{\tau}\right) \\
& =e^{\frac{2 \pi i x \omega}{\tau}} \frac{1}{\sqrt{\tau(1-\tau)}} e^{\frac{-2 \pi i x \omega}{\tau(1-\tau)}} e^{\frac{-2 \pi i(-x) \omega}{\tau(1-\tau)}}\left(D_{\frac{\tau}{\tau-1}}(g * k) * M_{\frac{-\omega}{\tau}} f_{2}^{*}\right)\left(\frac{-x}{1-\tau}\right) \\
& =\frac{1}{\sqrt{\tau(1-\tau)}} e^{\frac{2 \pi i x \omega(1+\tau)}{\tau(1-\tau)}} \overline{\left.\frac{\tau}{\tau-1}\right|^{\frac{-1}{2}}\left(\left(D_{\frac{\tau}{\tau-1}} g * D_{\frac{\tau}{\tau-1}} k\right) * M_{\frac{-\omega}{\tau}} f_{2}^{*}\right)\left(\frac{-x}{1-\tau}\right)} \\
& =\frac{1}{\tau} e^{\frac{2 \pi i x \omega(1+\tau)}{\tau(1-\tau)}} \overline{\left(D_{\frac{\tau}{\tau-1}} k *\left(D_{\frac{\tau}{\tau-1}} g * M_{\frac{-\omega}{\tau}} f_{2}^{*}\right)\right)\left(\frac{-x}{1-\tau}\right)} \text {. }
\end{aligned}
$$

Theorem 2.13. Suppose that $\omega_{3}=k_{1}$ and $\omega_{4}=k_{2}$ such that $k_{1}$ and $k_{2}$ are constant numbers. Then $C W_{\omega_{1}, \omega_{2}, \omega_{3}, \omega_{4}}^{p, q, r, s}(\mathbb{R})$ is an essential Banach module over $\left(L_{\omega_{1}}^{1} \times L_{\omega_{2}}^{1}\right)(\mathbb{R})$.

Proof. Let $\omega_{3}$ and $\omega_{4}$ be constant weight functions. It is known that $C W_{\omega_{1}, \omega_{2}, \omega_{3}, \omega_{4}}^{p, q, r, \tau}(\mathbb{R})$ is a Banach space by Theorem 2.2. Now we take any $(f, g) \in C W_{\omega_{1}, \omega_{2}, \omega_{3}, \omega_{4}}^{p, q, r, \omega_{1}}(\mathbb{R})$ and $(h, k) \in\left(L_{\omega_{1}}^{1} \times L_{\omega_{2}}^{1}\right)(\mathbb{R})$. Since $L_{\omega_{1}}^{p}(\mathbb{R})$ and $L_{\omega_{2}}^{q}(\mathbb{R})$ are Banach convolution module over $L_{\omega_{1}}^{1}(\mathbb{R})$ and $L_{\omega_{2}}^{1}(\mathbb{R})$ respectively, we have

$$
\|f * h\|_{p, \omega_{1}} \leq\|f\|_{p, \omega_{1}}\|h\|_{1, \omega_{1}}
$$


and

$$
\|g * k\|_{q, \omega_{2}} \leq\|g\|_{q, \omega_{2}}\|k\|_{1, \omega_{2}} .
$$

Take any $f_{1} \in S(\mathbb{R})$. By Theorem 2.12 , we get

$$
\begin{aligned}
& \left\|W_{\tau}\left(f * h, f_{1}\right)\right\|_{r, \omega_{3}}=\left\|\frac{1}{\sqrt{\tau(1-\tau)}} e^{\frac{-2 \pi i x \omega}{1-\tau}}\left(h *\left(f * M_{\frac{\omega}{\tau}}\left(D_{\frac{\tau}{\tau-1}} f_{1}\right)^{*}\right)\right)\left(\frac{x}{1-\tau}\right)\right\|_{r, \omega_{3}} \\
& =\frac{1}{\sqrt{\tau(1-\tau)}}\left\|\int_{\mathbb{R}} h(u) T_{u}\left(f * M_{\frac{\omega}{\tau}}\left(D_{\frac{\tau}{\tau-1}} f_{1}\right)^{*}\right)\left(\frac{x}{1-\tau}\right) d u\right\|_{r, \omega_{3}} \\
& =\frac{1}{\sqrt{\tau(1-\tau)}} \int_{\mathbb{R}}|h(u)|\left\|T_{u}\left(f * M_{\frac{\omega}{\tau}}\left(D_{\frac{\tau}{\tau-1}} f_{1}\right)^{*}\right)\left(\frac{x}{1-\tau}\right)\right\|_{r, \omega_{3}} d u \\
& =\frac{1}{\sqrt{\tau(1-\tau)}} \int_{\mathbb{R}}|h(u)|\left\|\left(f * M_{\frac{\omega}{\tau}}\left(D_{\frac{\tau}{\tau-1}} f_{1}\right)^{*}\right)\left(\frac{x}{1-\tau}\right)\right\|_{r, \omega_{3}} d u \\
& =\frac{1}{\sqrt{\tau(1-\tau)}} \int_{\mathbb{R}}|h(u)|\left\|e^{\frac{2 \pi i x \omega}{\tau(1-\tau)}} e^{\frac{-2 \pi i x \omega}{\tau(1-\tau)}}\left(f * M_{\frac{\omega}{\tau}}\left(D_{\frac{\tau}{\tau-1}} f_{1}\right)^{*}\right)\left(\frac{x}{1-\tau}\right)\right\|_{r, \omega_{3}} d u \\
& =\frac{1}{\sqrt{\tau(1-\tau)}} \int_{\mathbb{R}}|h(u)|\left\|e^{\frac{2 \pi i x \omega}{\tau(1-\tau)}} V_{D_{\bar{\tau}-1}} f_{1} f\left(\frac{x}{1-\tau}, \frac{\omega}{\tau}\right)\right\|_{r, \omega_{3}} d u \\
& =\frac{1}{\sqrt{\tau(1-\tau)}} \int_{\mathbb{R}}|h(u)|\left\|V_{D_{\tau-1}^{\tau} f_{1}}^{\tau} f(x, \omega)\right\|_{r, \omega_{3}} d u \\
& =\int_{\mathbb{R}}|h(u)|\left\|e^{\frac{-2 \pi i x \omega}{\tau}} \frac{1}{\sqrt{\tau(1-\tau)}} e^{\frac{2 \pi i x \omega}{\tau}} V_{D_{\frac{\tau}{\tau-1}}^{\tau} f_{1}} f(x, \omega)\right\|_{r, \omega_{3}} d u \\
& =\int_{\mathbb{R}}|h(u)|\left\|e^{\frac{-2 \pi i x \omega}{\tau}} W_{\tau}\left(f, f_{1}\right)\right\|_{r, \omega_{3}} d u \\
& =\left\|W_{\tau}\left(f, f_{1}\right)\right\|_{r, \omega_{3}}\|h\|_{1} \\
& \leq\left\|W_{\tau}\left(f, f_{1}\right)\right\|_{r, \omega_{3}}\|h\|_{1, \omega_{3}}<\infty .
\end{aligned}
$$

Thus $W_{\tau}(f * h,.) \in L_{\omega_{3}}^{r}\left(\mathbb{R}^{2}\right)$. Now take $f_{2} \in S(\mathbb{R})$. Again by Theorem 2.12, we have

$$
\begin{aligned}
\left\|W_{\tau}\left(f_{2}, g * k\right)\right\|_{s, \omega_{4}} & =\left\|\frac{1}{\tau} e^{\frac{2 \pi i x \omega(1+\tau)}{\tau(1-\tau)}} \overline{\left(D_{\bar{\tau}-1} k *\left(D_{\bar{\tau}-1} g * M_{\frac{-\omega}{\tau}} f_{2}^{*}\right)\right)\left(\frac{-x}{1-\tau}\right)}\right\|_{s, \omega_{4}} \\
& =\frac{1}{\tau}\left\|\left(D_{\frac{\tau}{\tau-1}} k *\left(D_{\frac{\tau}{\tau-1}} g * M_{\frac{-\omega}{\tau}} f_{2}^{*}\right)\right)\left(\frac{-x}{1-\tau}\right)\right\|_{s, \omega_{4}} \\
& =\frac{1}{\tau}\left\|\int_{\mathbb{R}} D_{\frac{\tau}{\tau-1}} k(u) T_{u}\left(D_{\frac{\tau}{\tau-1}} g * M_{\frac{-\omega}{\tau}} f_{2}^{*}\right)\left(\frac{-x}{1-\tau}\right) d u\right\|_{s, \omega_{4}} \\
& =\frac{1}{\tau} \int_{\mathbb{R}}\left\|D_{\frac{\tau}{\tau-1}} k(u) T_{u}\left(D_{\frac{\tau}{\tau-1}} g * M_{\frac{-\omega}{\tau}} f_{2}^{*}\right)\left(\frac{-x}{1-\tau}\right)\right\|_{s, \omega_{4}} d u
\end{aligned}
$$




$$
\begin{aligned}
& =\frac{1}{\tau} \int_{\mathbb{R}}\left|D_{\frac{\tau}{\tau-1}} k(u)\right|\left\|e^{\frac{2 \pi i x \omega}{\tau(1-\tau)}} e^{\frac{-2 \pi i x \omega}{\tau(1-\tau)}}\left(D_{\frac{\tau}{\tau-1}} g * M_{\frac{-\omega}{\tau}} f_{2}^{*}\right)\left(\frac{-x}{1-\tau}\right)\right\|_{s, \omega_{4}} d u \\
& =\frac{1}{\tau} \int_{\mathbb{R}}\left|D_{\frac{\tau}{\tau-1}} k(u)\right|\left\|e^{\frac{2 \pi i x \omega}{\tau(1-\tau)}} V_{f_{2}} D_{\frac{\tau}{\tau-1}} g\left(\frac{-x}{1-\tau}, \frac{-\omega}{\tau}\right)\right\|_{s, \omega_{4}} d u \\
& =\frac{1}{\tau} \int_{\mathbb{R}}\left|D_{\frac{\tau}{\tau-1}} k(u)\right|\left\|e^{\frac{4 \pi i x \omega}{\tau(1-\tau)}} e^{\frac{-2 \pi i x \omega}{\tau(1-\tau)}} V_{f_{2}} D_{\frac{\tau}{\tau-1}} g\left(\frac{-x}{1-\tau}, \frac{-\omega}{\tau}\right)\right\|_{s, \omega_{4}} d u \\
& =\frac{1}{\tau} \int_{\mathbb{R}}\left|D_{\frac{\tau}{\tau-1}} k(u)\right|\left\|e^{\frac{4 \pi i x \omega}{\tau(1-\tau)}} V_{D_{\frac{\tau}{\tau-1}}} f_{2}\left(\frac{x}{1-\tau}, \frac{\omega}{\tau}\right)\right\|_{s, \omega_{4}} d u \\
& =\frac{1}{\tau} \int_{\mathbb{R}}\left|D_{\frac{\tau}{\tau-1}} k(u)\right|\left\|\sqrt{\tau(1-\tau)} e^{\frac{-2 \pi i x \omega}{\tau}} \frac{1}{\sqrt{\tau(1-\tau)}} e^{\frac{2 \pi i x \omega}{\tau}} V_{D_{\bar{\tau}}^{\tau} \tau} g f_{2}(x, \omega)\right\|_{s, \omega_{4}} d u \\
& =\frac{1}{\tau} \sqrt{\tau(1-\tau)} \int\left|D_{\frac{\tau}{\tau-1}} k(u)\right|\left\|W_{\tau}\left(f_{2}, g\right)\right\|_{s, \omega_{4}} d u \\
& =\sqrt{\frac{1-\tau}{\tau}}\left\|W_{\tau}\left(f_{2}, g\right)\right\|_{s, \omega_{4}}\left\|D_{\frac{\tau}{\tau-1}} k\right\|_{1} \\
& =\sqrt{\frac{1-\tau}{\tau}}\left\|W_{\tau}\left(f_{2}, g\right)\right\|_{s, \omega_{4}} \sqrt{\frac{\tau}{1-\tau}}\|k\|_{1}
\end{aligned}
$$

$$
\leq\left\|W_{\tau}\left(f_{2}, g\right)\right\|_{s, \omega_{4}}\|k\|_{1, \omega_{4}}<\infty .
$$

So $W_{\tau}(., g * k) \in L_{\omega_{4}}^{s}\left(\mathbb{R}^{2}\right)$. Combining (2.12), (2.13), (2.14) and (2.15), we achieve

$$
\begin{aligned}
& \|(f, g) *(h, k)\|_{C W_{\omega_{1}, \omega_{2}, \omega_{3}, \omega_{4}}^{p, q, r, \tau}}=\|(f * h, g * k)\|_{C W_{\omega_{1}, \omega_{2}, \omega_{3}, \omega_{4}}^{p, q, s,}}= \\
& =\|(f * h, g * k)\|_{L_{\omega_{1}}^{p} \times L_{\omega_{2}}^{q}}+\left\|\left(W_{\tau}(f * h, .), W_{\tau}(., g * k)\right)\right\|_{L_{\omega_{3}}^{r} \times L_{\omega_{4}}^{s}} \\
& =\max \left\{\|f * h\|_{p, \omega_{1}},\|g * k\|_{q, \omega_{2}}\right\}+\max \left\{\left\|W_{\tau}(f * h, .)\right\|_{r, \omega_{3}},\left\|W_{\tau}(., g * k)\right\|_{s, \omega_{4}}\right\} \\
& =\max \left\{\|f\|_{p, \omega_{1}}\|h\|_{1, \omega_{1}},\|g\|_{q, \omega_{2}}\|k\|_{1, \omega_{2}}\right\}+\max \left\{\left\|W_{\tau}(f, .)\right\|_{r, \omega_{3}}\|h\|_{1, \omega_{3}},\left\|W_{\tau}(., g)\right\|_{s, \omega_{4}}\|k\|_{1, \omega_{4}}\right\} \\
& =\max \left\{\|f\|_{p, \omega_{1}},\|g\|_{q, \omega_{2}}\right\} \max \left\{\|h\|_{1, \omega_{1}},\|k\|_{1, \omega_{2}}\right\}+ \\
& +\max \left\{\left\|W_{\tau}(f, .)\right\|_{r, \omega_{3}},\left\|W_{\tau}(., g)\right\|_{s, \omega_{4}}\right\} \max \left\{\|h\|_{1, \omega_{3}},\|k\|_{1, \omega_{4}}\right\} \\
& =\max \left\{\|f\|_{p, \omega_{1}},\|g\|_{q, \omega_{2}}\right\} \max \left\{\|h\|_{1, \omega_{1}},\|k\|_{1, \omega_{2}}\right\}+ \\
& =\max \left\{\left\|W_{\tau}(f, .)\right\|_{r, \omega_{3}},\left\|W_{\tau}(., g)\right\|_{s, \omega_{4}}\right\} \max \left\{\|h\|_{1, \omega_{1}},\|k\|_{1, \omega_{2}}\right\} \\
& =\left\{\|(f, g)\|_{L_{\omega_{1}}^{p} \times L_{\omega_{2}}^{q}}+\left\|\left(W_{\tau}(f, .), W_{\tau}(., g)\right)\right\|_{L_{\omega_{3}}^{r} \times L_{\omega_{4}}^{s}}\right\}\|(h, k)\|_{L_{\omega_{1}}^{1} \times L_{\omega_{2}}^{1}} \\
& (2.16) \\
& =\|(f, g)\|_{C W_{\omega_{1}, \omega_{2}, \omega_{3}, \omega_{4}}^{p, q, r, s}}\|(h, k)\|_{L_{\omega_{1}}^{1} \times L_{\omega_{2}}^{1}} .
\end{aligned}
$$

Therefore we obtain that $C W_{\omega_{1}, \omega_{2}, \omega_{3}, \omega_{4}}^{p, q, s, \tau}(\mathbb{R})$ is a Banach module over $\left(L_{\omega_{1}}^{1} \times L_{\omega_{2}}^{1}\right)(\mathbb{R})$. 
Now we will show that $C W_{\omega_{1}, \omega_{2}, \omega_{3}, \omega_{4}}^{p, q, r, \tau}(\mathbb{R})$ is an essential Banach module over $\left(L_{\omega_{1}}^{1} \times L_{\omega_{2}}^{1}\right)(\mathbb{R})$ and use Module Factorization Theorem [12]. For this, it suffices to prove that $\left(L_{\omega_{1}}^{1} \times L_{\omega_{2}}^{1}\right)(\mathbb{R}) * C W_{\omega_{1}, \omega_{2}, \omega_{3}, \omega_{4}}^{p, q, r, \tau}(\mathbb{R})$ is dense in $C W_{\omega_{1}, \omega_{2}, \omega_{3}, \omega_{4}}^{p, q, r, s, \tau}(\mathbb{R})$. By (2.16), we can write

$$
\left(L_{\omega_{1}}^{1} \times L_{\omega_{2}}^{1}\right)(\mathbb{R}) * C W_{\omega_{1}, \omega_{2}, \omega_{3}, \omega_{4}}^{p, q, s, \tau}(\mathbb{R}) \subset C W_{\omega_{1}, \omega_{2}, \omega_{3}, \omega_{4}}^{p, q, r, s}(\mathbb{R}) .
$$

Also it is known that $L_{\omega_{1}}^{1}(\mathbb{R})$ and $L_{\omega_{2}}^{1}(\mathbb{R})$ have bounded approximate identity, [6]. Let $U$ and $V$ be compact neighbourhoods of the unit element of $\mathbb{R}$. We can choose approximate identities $\left(e_{\alpha}\right)_{\alpha \in I}$ and $\left(e_{\beta}\right)_{\beta \in I}$ which are positive bounded and suppe $_{\alpha} \subset U$, suppe $_{\beta} \subset V,\left\|e_{\alpha}\right\|_{1}=1$ and $\left\|e_{\beta}\right\|_{1}=1$ for all $\alpha, \beta \in I$. Let $(h, k) \in C W_{\omega_{1}, \omega_{2}, \omega_{3}, \omega_{4}}^{p, q, r, \tau}(\mathbb{R})$. For fixed $\alpha_{0}, \beta_{0} \in I$, we get

$$
\begin{aligned}
\|\left(e_{\alpha_{0}}, e_{\beta_{0}}\right) *(h, k) & -(h, k)\left\|_{C W_{\omega_{1}, \omega_{2}, \omega_{3}, \omega_{4}}^{p, q, r, s, \tau}}=\right\|\left(e_{\alpha_{0}} * h-h, e_{\beta_{0}} * k-k\right) \|_{C W_{\omega_{1}, \omega_{2}, \omega_{3}, \omega_{4}}^{p, q, r, s, \tau}} \\
& \approx\left\|\left(e_{\alpha_{0}} * h-h, e_{\beta_{0}} * k-k\right)\right\|_{C W_{\omega_{1}, \omega_{3}}^{p, r, \tau} \times C W_{\omega_{2}, \omega_{4}}^{q, s, \tau}} \\
& =\left\|e_{\alpha_{0}} * h-h\right\|_{C W_{\omega_{1}, \omega_{3}}^{p, r, \tau}}+\left\|e_{\beta_{0}} * k-k\right\|_{C W_{\omega_{2}, \omega_{4}}^{q, s, \tau}} .
\end{aligned}
$$

On the other hand, since the translation mapping is continuous by Corollary 1, we have $\left\|T_{z} h-h\right\|_{C W_{\omega_{1}, \omega_{3}}^{p, r, \tau}}<\frac{\varepsilon}{2}$ for given any $\varepsilon>0$. Hence

$$
\begin{aligned}
\left\|e_{\alpha_{0}} * h-h\right\|_{C W_{\omega_{1}, \omega_{3}}^{p, r, \tau}} & =\left\|\int_{\mathbb{R}} e_{\alpha_{0}}(z)\left(T_{z} h(y)-h(y)\right) d z\right\|_{C W_{\omega_{1}, \omega_{3}}^{p, r, \tau}} \\
& \leq \int_{\mathbb{R}^{d}} e_{\alpha_{0}}(z)\left\|T_{z} h-h\right\|_{C W_{\omega_{1}, \omega_{3}}^{p, r, \tau}} d z<\frac{\varepsilon}{2} .
\end{aligned}
$$

Similarly we write for the same $\varepsilon>0$, we can make

$$
\left\|e_{\beta_{0}} * k-k\right\|_{C W_{\omega_{2}, \omega_{4}}^{q, s, \tau}}<\frac{\varepsilon}{2} .
$$

Then, by (2.17), (2.18) and (2.19), we obtain

$$
\left\|\left(e_{\alpha_{0}}, e_{\beta_{0}}\right) *(h, k)-(h, k)\right\|_{C W_{\omega_{1}, \omega_{2}, \omega_{3}, \omega_{4}}^{p, q, r, \tau}}<\frac{\varepsilon}{2}+\frac{\varepsilon}{2}=\varepsilon .
$$

That means $\left(L_{\omega_{1}}^{1} \times L_{\omega_{2}}^{1}\right)(\mathbb{R}) * C W_{\omega_{1}, \omega_{2}, \omega_{3}, \omega_{4}}^{p, q, r, \tau}(\mathbb{R})$ is dense in $C W_{\omega_{1}, \omega_{2}, \omega_{3}, \omega_{4}}^{p, q, r, \tau}(\mathbb{R})$. Therefore from Module Factorization Theorem, the proof is completed.

By using Theorem 6, it easy to prove following Corollary

Corollary 2. Assume that $\omega_{3}=k_{1}$ and $\omega_{4}=k_{2}$ such that $k_{1}$ and $k_{2}$ are constant numbers. Then,

a) $C W_{\omega_{1}, \omega_{3}}^{p, r, \tau}(\mathbb{R})$ is an essential Banach module over $L_{\omega_{1}}^{1}(\mathbb{R})$.

b) $C W_{\omega_{2}, \omega_{4}}^{q, s, \tau}(\mathbb{R})$ is an essential Banach module over $L_{\omega_{2}}^{1}(\mathbb{R})$.

Theorem 2.14. Let $\omega_{3}=k_{1}$ and $\omega_{4}=k_{2}$ be constant weight functions. Then there exists $\left(\left(e_{\alpha}, e_{\beta}\right)\right)_{\alpha, \beta \in I}$ is an approximate identity of the space $\left(L_{\omega_{1}}^{1} \times L_{\omega_{2}}^{1}\right)(\mathbb{R})$ such that

$$
\lim _{\alpha, \beta \in I}\left(e_{\alpha}, e_{\beta}\right) *(f, g)=(f, g)
$$

for all $(f, g) \in C W_{\omega_{1}, \omega_{2}, \omega_{3}, \omega_{4}}^{p, q, r, s, \tau}(\mathbb{R})$. 
Proof. Using the fact that $L_{\omega_{1}}^{1}(\mathbb{R})$ and $L_{\omega_{2}}^{1}(\mathbb{R})$ have bounded approximate identities $\left(e_{\alpha}\right)_{\alpha \in I}$ and $\left(e_{\beta}\right)_{\beta \in I}$, respectively, we easily obtain that $\left(\left(e_{\alpha}, e_{\beta}\right)\right)_{\alpha, \beta \in I}$ is an approximate identity in $\left(L_{\omega_{1}}^{1} \times L_{\omega_{2}}^{1}\right)(\mathbb{R})$. On the other hand, from Corollary 2 and by [3], we get

$$
\lim _{\alpha \in I} e_{\alpha} * f=f, \lim _{\beta \in I} e_{\beta} * g=g
$$

for all $f \in C W_{\omega_{1}, \omega_{3}}^{p, r, \tau}(\mathbb{R})$ and $g \in C W_{\omega_{2}, \omega_{4}}^{q, s, \tau}(\mathbb{R})$. Therefore by (2.20), we obtain

$$
\lim _{\alpha, \beta \in I}\left(e_{\alpha}, e_{\beta}\right) *(f, g)=\lim _{\alpha, \beta \in I}\left(e_{\alpha} * f, e_{\beta} * g\right)=(f, g)
$$

$(f, g) \in C W_{\omega_{1}, \omega_{2}, \omega_{3}, \omega_{4}}^{p, q, r, \tau}(\mathbb{R})$.

\section{Conclusion}

Wigner transform, which is a quadratic time-frequency representation; it is very ideal in the mathematical description of the time-frequency information of the signals. The reasons for its preference can be summarized as follows: The Wigner transform measures the energy of a given signal in any frequency band and in any time period. Thus, in signal analysis, the information of the energy density in the time-frequency plane is obtained. It also gives the joint probability distribution for position and momentum variables in physics, [7]. In our previous papers, we have characterized function spaces using the wavelet transform and fractional wavelet transform, $[4,8]$. In this study, we defined a new function space using the $\tau$-Wigner transform, which is a quadric time-frequency transform. We then have studied the Banach module structure of this space, the continuity of the translation mapping and its approximate units. In this way, a new function space with rich features was characterized thanks to the $\tau$-Wigner transform, which is frequently used in harmonic analysis, signal analysis and operator theory.

\section{Acknowledgments}

The authors would like to thank the reviewers and editors of Journal of Universal Mathematics.

\section{Funding}

The first author was supported by Giresun University, Project number FEN-BAPC-150219-01.

The Declaration of Conflict of Interest/ Common Interest

The author(s) declared that no conflict of interest or common interest

\section{The Declaration of Ethics Committee Approval}

This study does not be necessary ethical committee permission or any special permission.

\section{The Declaration of Research and Publication Ethics}

The author(s) declared that they comply with the scientific, ethical, and citation rules of Journal of Universal Mathematics in all processes of the study and that they do not make any falsification on the data collected. Besides, the author(s) 
declared that Journal of Universal Mathematics and its editorial board have no responsibility for any ethical violations that may be encountered and this study has not been evaluated in any academic publication environment other than Journal of Universal Mathematics.

\section{REFERENCES}

[1] P. Boggiatto, G. De Donno, A. Oliaro, A class of quadratic time- frequency representations based on the short- time Fourier transform, Oper Theory, 172, 235-249, (2007).

[2] P. Boggiatto, G. De Donno, A. Oliaro, Time- frequency representations of Wigner type and pseudo- differential operators, Trans Amer Math Soc, 362, 4955-4981, (2010).

[3] R.S. Doran, J. Wichmann, Approximate identity and factorization in Banach modules, Lecture Notes in Math. Springer-Verlag, 768 (1979).

[4] M. Duman, Ö. Kulak, On Function Spaces with Fractional Wavelet Transform, Montes Taurus J. Pure Appl. Math. 3 (3), 122-134 (2021).

[5] R.H. Fischer, A.T. Gürkanl, T.S. Liu, On a family of weighted spaces, Mathematica Slovaca, 46(1), 71-82 (1996).

[6] I.G. Gaudry, Multipliers of weighted Lebesgue and measure spaces, Proc.Lon.Math.Soc., 19(3), 327-340 (1969).

[7] K. Gröchenig, Foundations of Time-Frequency Analysis, Birkhauser, Boston (2001).

[8] Ö. Kulak, A.T. Gürkanl, On Function Spaces with Wavelet Transform in $L_{\omega}^{p}\left(\mathbb{R}^{d} \times \mathbb{R}_{+}\right)$, Hacettepe Journal of Mathematics and Statistics, 40(2), 163-177 (2011).

[9] H. Reiter, Classical Harmonic Analysis and Locally Compact Group, Oxford Universty Pres, Oxford (1968).

[10] A. Sandıkçı, Continuity of Wigner-type operators on Lorentz spaces and Lorentz mixed normed modulation spaces, Turkish Journal of Mathematics, 38, 728- 745 (2014).

[11] A. Sandıkçı, Multilinear $\tau$-Wigner transform, J. Pseudo-Differ. Oper. Appl., 11, 1465-1487 (2020).

[12] H.C. Wang, Homogeneous Banach algebras, New York: Marcel Dekker Inc. (1977).

Amasya University, Faculty of Arts and Sciences, Department of Mathematics, 05100, AMASYA, TURKEY

Email address: oznur.kulak@amasya.edu.tr

Giresun University, Faculty of Arts and Sciences, Department of Mathematics, 28200, Giresun, TURKEY

Email address: yunusfatel@gmail.com 